\title{
Social Activity Patterns Drive High Rates of Latent Tuberculosis Infection among Adolescents in Urban Tanzania
}

\section{- Latent TB Infection in Adolescents, Tanzania}

\author{
Isaac I. Maro' ${ }^{1}$ Keiko Nakamura ${ }^{*}$, Kaoruko Seino' ${ }^{1}$, Kisali Pallangyo², Patricia Munseri ${ }^{3}$, \\ Mecky Matee ${ }^{4}$, Charles Fordham von Reyn ${ }^{5}$
}

${ }^{1}$ Department of Global Health Entrepreneurship, Tokyo Medical and Dental University, Tokyo, Japan

${ }^{2}$ Muhimbili University of Health and Allied Sciences, Dar es Salaam, Tanzania

${ }^{3}$ Department of Internal Medicine, Muhimbili University of Health and Allied Sciences, Dar es Salaam,

Tanzania

${ }^{4}$ Department of Microbiology and Immunology, School of Medicine, Muhimbili University of Health and Allied Sciences,

Dar es Salaam, Tanzania

${ }^{5}$ Geisel School of Medicine at Dartmouth, Hanover, NH, USA

Email: *nakamura.ith@tmd.ac.jp

How to cite this paper: Maro, I.I., Nakamura, K., Seino, K., Pallangyo, K., Munseri, P., Matee, M. and von Reyn, C.F. (2018) Social Activity Patterns Drive High Rates of Latent Tuberculosis Infection among Adolescents in Urban Tanzania. Journal of Tuberculosis Research, 6, 81-95. https://doi.org/10.4236/jtr.2018.61008

Received: January 26, 2018

Accepted: March 23, 2018

Published: March 26, 2018

Copyright ( 92018 by authors and Scientific Research Publishing Inc. This work is licensed under the Creative Commons Attribution International License (CC BY 4.0).

http://creativecommons.org/licenses/by/4.0/

\section{cc) (i) Open Access}

\begin{abstract}
SETTING: Dar es Salaam, Tanzania. OBJECTIVE: To determine the prevalence of latent tuberculosis (TB) infection (LTBI) among adolescents in a country with a high TB burden, and examine risks of LTBI according to their social activity patterns. METHODS: A cross-sectional study nested within a phase $2 \mathrm{~b}$ randomised, placebo controlled, double blind study and consisted of 824 adolescents, 13 - 15 years old who had received Bacillus Calmette-Guérin (BCG) vaccine, were attending public secondary schools and had no evidence of active tuberculosis (TB). Anthropometric measurements were obtained, a questionnaire administered, and phlebotomy performed for a $\mathrm{T}$ spot interferon- $\gamma$ release assay (IGRA) to detect LTBI. RESULTS: Among 824 subjects, 149 (18\%) had a positive IGRA. After adjusting for the influence of household socioeconomic status, history of TB contact, living environment and nutritional status, LTBI risk was higher in subjects with than without regular informal encounters with traditional alcoholic beverage drinkers (AOR, 6.37 [1.84 - 22.00]). Other significant factors for LTBI risk included contact with TB patient at school (AOR, 3.34 [1.14 - 9.80]), and living close to a health facility, as was observed among those from houses within a 10 - 30-minute walking distance to the nearest health facility, who were less likely to be IGRA-positive than those who were living within a 10-minute walking dis-
\end{abstract}


tance (AOR, 0.30 [95\%CI, 0.13 - 0.69]). CONCLUSION: This IGRA study revealed a high prevalence of LTBI among adolescents in Dar es Salaam, Tanzania with prior BCG immunization. Informal social encounters were identified as independent risk factors for LTBI, along with a history of contact with TB patients, living environment characteristics and household socioeconomic status. Efforts focusing on risk of MTB transmission in adolescents at informal social gatherings will improve interventions to reduce LTBI in this population and consequently the subsequent risk of developing active TB disease.

\section{Keywords}

Dar es Salaam, Social Factors, IGRA, T Spot, Adolescents, BCG, DAR 901

\section{Introduction}

Despite a decline in its global prevalence, tuberculosis (TB) still causes more deaths than other infectious diseases [1]. In 2015, 10.4 million new cases of TB and 1.4 million deaths from TB were reported globally [1]. Twenty countries, including Tanzania, contribute $84 \%$ of total global TB cases [1].

About one third of the world's population is infected with the causative agent of TB, Mycobacterium tuberculosis (MTB) [2], but most show no signs or symptoms of active disease, i.e., latent TB infection (LTBI) [2]. Approximately $10 \%$ of those with LTBI will progress to active TB at some point in their lives [2] [3]. Infant BCG immunization is $>70 \%$ effective in preventing TB disease for 10 - 15 years [4] [5], after which the level of protection begins to wane [5] [6]. In Tanzania, where TB is endemic, and $91 \%$ of all children $<12$ months of age have received the Bacillus Calmette-Guerin (BCG) vaccine against $\mathrm{TB}$, the annual incidence of TB disease in 2015 was 306/100,000, including 18,000 children $<14$ years of age [1] [7].

Data on the prevalence of LTBI and risk factors for LTBI in Tanzania are more limited, and are not available for adolescents. This is true despite the high incidence of TB disease among children $<14$ years old in Tanzania [1], the waning efficacy of BCG vaccine in adolescence [5] [6], the adolescents' increased outdoor activities [8], 43.9\% of country population in 2012 being children $\leq 15$ years [9], and the rapid increase in urban populations in Tanzania [9].

The $\mathrm{T}$ spot interferon- $\gamma$ release assay (IGRA) is an advanced TB diagnostic tool, and is more specific than the tuberculin skin testing (TST) in identifying LTBI among BCG-vaccinated individual [10] [11]. Risk factors for LTBI reported from other settings include household contact with infectious TB [12] [13], overcrowding and poor ventilation within households [14] [15], HIV infection [16], underweight [16] and high TB incidence [17]. Most of these studies have been conducted in settings where BCG immunization is routine and have used the TST to identify LTBI.

In this study, we used IGRA as the most specific approach to detect LTBI in 
adolescents at school, who had received BCG at birth. Our objectives were to obtain an accurate estimate of the prevalence of LTBI in adolescents, and to identify risk factors for LTBI with a focus on both the home environment and social encounters outside the home.

\section{Methods}

\subsection{Study Procedure}

This study was conducted between April 6 and September 23, 2016, in Dar es Salaam, Tanzania, with a population of 5,465,420 as of 2016 [9]. This was part of a larger phase $2 \mathrm{~b}$, randomised, placebo-controlled, double-blind study on the safety and efficacy of a three-dose series of DAR-901 an inactivated whole cell vaccine for preventing MTB infection among BCG-vaccinated adolescents [1] [18] [19]. Written informed consent and assent from parents/guardians and study subjects were obtained. Anthropometric measurements and phlebotomy were performed for IGRA, and subjects completed a questionnaire.

\subsection{Study Subjects}

Subjects were recruited from 16 secondary schools chosen from a list of 139 public non-boarding secondary schools within the Dar es Salaam region [20]. These 16 schools were selected by simple random selection, evaluation of accessibility to a study clinic and willingness of the school administration to participate in the study. Based on the LTBI prevalence rate of $41.7 \%$ among adolescents 11 - 15 years old reported in South Africa [21], and the difference in reported incidences of TB in Tanzania and South Africa, with 5\% type I error and 5\% absolute error, we calculated that our study required a sample size of 571 .

Initial screening for the study was performed after obtaining written informed consent and assent from a total of 936 subjects. The inclusion criteria were: age between 13 and 15 years, attendance at a public non-boarding secondary school, evidence of BCG vaccination (either the presence of a scar or documentation) and the absence of signs of active TB or symptoms suggesting active TB disease based on the Tanzanian TB screening questionnaire [22]. Data from 824 subjects that fulfilled the inclusion criteria were analysed. Those with signs and/or symptoms of active TB were referred to specialised TB clinics under the National Tuberculosis and Leprosy Program (NTLP).

\subsection{Anthropometric Measurements}

Body mass index (BMI) $\left(\mathrm{kg} / \mathrm{m}^{2}\right)$ was calculated based on height and weight measurements. BMI-for-age percentiles for both genders, obtained using the Centres for Disease Control (CDC) growth charts [23], were categorised as follows: $<5 \%$, underweight; $5 \%$ - $85 \%$, healthy; $85 \%$ - 95\%, overweight; $>95 \%$, obese.

\subsection{Interferon- $\gamma$ Release Assay (IGRA)}

The IGRA was performed using the T-SPOT.TB (Oxford Immunotec, Oxford, 
UK) assay according to the manufacturer's instructions. Venous blood samples were analysed at the Tuberculosis Research Institute at Muhimbili University of Health and Allied Sciences (TRIM-TB) in Dar es Salaam, Tanzania [18]. The results were interpreted as positive, negative, borderline or indeterminate according to the published guidelines [18]. Adolescents with positive results were administered the Tanzanian TB screening questionnaire [22] and referred to specialised NTLP TB treatment clinics if signs or symptoms consistent with active TB were present [18].

\subsection{A TB Risk Factor Questionnaire}

A questionnaire was developed at the Department of Global Health Entrepreneurship of Tokyo Medical and Dental University, and included questions about prior active $\mathrm{TB}$, contact with $\mathrm{TB}$ patients, social activity patterns, living environment and household socioeconomic characteristics. The original English version questionnaire was translated into Swahili, which was then back translated to English for confirmation.

After receiving instructions on how to complete the questionnaire, the adolescents were allowed to take the questionnaire home to answer all questions with the help of their parents/guardians. At the time of submission, nurses inspected the completed questionnaires to confirm that they had been filled out correctly. To ensure consistency in instruction and interviews, all nurses involved in the study received a full day of training before initiation of the study.

We obtained the subject's past history of diagnosis and treatment for active TB disease, household contact with a TB patient, knowledge of a neighbour that had TB, and knowledge of a fellow student diagnosed with TB.

To understand the social activity patterns of the adolescents, the questionnaire inquired about the means of transport used to attend school, encounters with traditional alcoholic beverage drinkers, visits to market places and walking distance between the house and the nearest health facility. All these activities increase risks for contact with active TB patients.

In relation to the living environment characteristics, the number of living rooms and bedrooms, brightness from sunlight in living rooms and bedrooms and number of older family members living in the same house were determined.

The education levels of the father and mother, employment of father and household wealth status evaluated according to the Demographic and Health Survey (DHS) guidelines [24] [25] were used.

\subsection{Statistical Analysis}

Statistical analyses were performed using SPSS Statistics v22.0 (IBM Corp., Armonk, NY, USA). Percentage distributions according to IGRA results were calculated for 824 subjects. After excluding 20 subjects with indeterminate and borderline results, the percentages of IGRA-positive cases according to their characteristics were evaluated using cross-tabulation with the $\chi^{2}$ test. After ex- 
cluding 75 subjects with prior history of TB diagnosis (23 who responded "Yes" and 52 who responded "I do not remember"), logistic regression analysis to examine the association between IGRA positivity and characteristics of the subjects were performed with three models: 1) crude; 2) adjusted by wealth, history of contact with TB patients in their households and in the community; and 3) by wealth, history of contact with TB patients in their households, in the community, living conditions and nutritional status. All missing data were excluded from multiple logistic regression analyses models. In all analyses, $P<0.05$ indicated statistical significance.

\subsection{Ethical Consideration}

This study was approved by the Institution Review Boards of the Medical Schools of Tokyo Medical and Dental University, Japan; Muhimbili University of Health and Allied Sciences, Tanzania; and Geisel School of Medicine at Dartmouth, NH, USA. Permission to recruit secondary school students was obtained from the Ministry of Health, Community Development, Gender, Elderly and Children, the Ministry of Education and Vocational Training, and the President's Office for Regional Administration and Local Government of Tanzania.

\section{Results}

Table 1 shows the results of IGRA; $18.1 \%$ of the 824 subjects were positive and $79.5 \%$ were negative.

Table 2 shows the number and percentage of the IGRA-positive cases according to the characteristics for selected variables showing statistically significant associations with the IGRA results.

Table 3 shows the associations between IGRA status and characteristics of 729 adolescents with no history of prior TB, before and after adjusting for other variables. The rate of IGRA positivity was higher in subjects with than without frequent encounters with traditional alcohol beverage drinkers (AOR, 6.37 [1.84 - 22.00]), and those residing in houses within a 10 - 30-minute walking distance to the nearest health facility were less likely to be IGRA-positive than those living

Table 1. IGRA results among adolescents $(n=824)$.

\begin{tabular}{ccc}
\hline CATEGORY & $\boldsymbol{n}$ & $\%$ \\
\hline IGRA Result & 149 & \\
\hline Positive & 655 & 18.1 \\
Negative & 19 & 79.5 \\
Indeterminate & 1 & 2.3 \\
Borderline & 824 & 0.1 \\
Total & & 100.0 \\
\hline
\end{tabular}

n, number of subjects; \%, percentage. 
Table 2. IGRA-positive cases according to adolescents' characteristics ( $n=804$, after excluding subjects with indeterminate or borderline test results).

\begin{tabular}{|c|c|c|c|c|}
\hline \multirow{2}{*}{$N=804$} & \multicolumn{4}{|c|}{ IGRA-positive adolescents } \\
\hline & $\%$ & $\mathbf{n}$ & $\%$ & \\
\hline
\end{tabular}

\section{Gender and nutritional status}

Gender

358

44.5
55.4

73

20.4

76

17.0

$$
\begin{aligned}
& \text { Boys } \\
& \text { Girls }
\end{aligned}
$$

Body Mass Index (BMI) percentile

$$
\begin{aligned}
& <5 \% \text { [underweight] } \\
& 5 \%-85 \% \text { [healthy] } \\
& 85 \%-95 \% \text { [overweight] } \\
& >95 \% \text { [obese] }
\end{aligned}
$$

History of being treated for TB

$$
\begin{aligned}
& \text { No } \\
& \text { Yes } \\
& \text { Unknown }
\end{aligned}
$$

Contact with TB patient(s) in household

$$
\begin{aligned}
& \text { No } \\
& \text { Yes } \\
& \text { Unknown }
\end{aligned}
$$

Contact with TB patient(s) in community

$$
\begin{aligned}
& \text { No } \\
& \text { Yes } \\
& \text { Unknown }
\end{aligned}
$$

Contact with TB patient(s) at school
No
Yes
Unknown

23

591

124

66

History of TB and contact with TB patients
0.12

0.01

$\begin{array}{lccc}729 & 90.7 & 132 & 18.1 \\ 23 & 2.9 & 9 & 39.1 \\ 52 & 6.5 & & \end{array}$

0.03

$\begin{array}{llll}616 & 76.6 & 100 & 16.2 \\ 90 & 11.2 & 23 & 25.6 \\ 98 & 12.2 & & \end{array}$

$<0.01$

$\begin{array}{llll}493 & 61.3 & 83 & 16.8 \\ 59 & 7.3 & 25 & 42.4\end{array}$

$252 \quad 31.3$

$<0.01$

$\begin{array}{llll}663 & 82.5 & 111 & 16.7 \\ 34 & 4.2 & 14 & 41.2\end{array}$

$107 \quad 13.3$

Social activity patterns among adolescents

Means of transport to and from school

0.04
Public transport
Walking
Bicycle

$\begin{array}{llll}308 & 38.3 & 46 & 14.9 \\ 476 & 59.2 & 99 & 20.8 \\ 20 & 2.5 & 5 & 25.0\end{array}$




\section{Continued}

Encounter with traditional alcoholic beverage drinkers

No, never

Yes, only few times in the past

Yes, once to a few times monthly

Yes, once a week or more frequently

Unknown

563

142

41

32

26

Visits to market places

Once or less per month

More than once per month

Unknown

Closeness to health facility

$<10$ minutes

$10-30$ minutes

$>30$ minutes

Unknown

286

197

96

225

Living environments

Number of living room(s)
0
1
2
Unknown

Brightness from sunlight in living room(s)

Lack of sunlight

Somewhat bright

Very bright

Unknown

Number of bedrooms(s)

1

2

$\geq 4$

Unknown

411

360

3
10

268

283

243

13

438

222

131

134

354

94

100

4.1

51.1

4.8

35.6

24.5

11.9

28.0
63

82

22.8

0.01

5.3

1

$\begin{array}{ll}68 & 23.8 \\ 27 & 13.7 \\ 17 & 17.7\end{array}$




\section{Continued}

Brightness from sunlight in bedroom(s)

Lack of sunlight at all
Somewhat bright
Very bright
Unknown

Family members older than adolescent

1

2

$\geq 3$

Unknown

Father's educational attainment

No
Primary education
Higher education
Unknown

Mother's educational attainment

No
Primary education
Higher education
Unknown

Father's employment

Unemployed
Businessman
Works in the office
Industrial worker
Unknown

Wealth status of household

Richest
Rich
Middle
Poor
Poorest

$\begin{array}{cccc}21 & 2.6 & 3 & 14.3 \\ 540 & 67.2 & 113 & 20.9 \\ 154 & 19.2 & 16 & 10.4 \\ 89 & 11.0 & & \end{array}$

0.04

$\begin{array}{rrrr}61 & 7.6 & 4 & 6.6 \\ 138 & 17.2 & 31 & 22.5 \\ 535 & 66.5 & 99 & 18.5 \\ 70 & & & \end{array}$

\section{Household socioeconomic characteristics}

$<0.01$

$\begin{array}{llll}21 & 2.6 & 12 & 57.1 \\ 288 & 35.8 & 69 & 24.0 \\ 305 & 37.9 & 43 & 14.1\end{array}$

$190 \quad 23.6$

0.03
17

370

238

179

22.3

2.1

46.0

29.6
29

448

95

39

193
35

14.7

$$
3.6
$$$$
55.7
$$$$
11.8
$$$$
4.9
$$$$
24.0
$$

24.0

.


Table 3. Association between IGRA positivity and characteristics of adolescents before and after adjustment for socioeconomic status, household and community TB contact, living conditions and nutritional status.

\begin{tabular}{|c|c|c|c|c|c|c|c|}
\hline \multirow{2}{*}{ Characteristics } & \multirow{2}{*}{$\mathrm{n}$} & MODEL $1^{\mathrm{a}}$ & \multirow{2}{*}{$P$} & MODEL $2^{\mathrm{b}}$ & \multirow{2}{*}{$P$} & MODEL $3^{c}$ & \multirow{2}{*}{$P$} \\
\hline & & COR $[95 \% \mathrm{CI}]$ & & AOR $[95 \% \mathrm{CI}]$ & & AOR $[95 \% \mathrm{CI}]$ & \\
\hline
\end{tabular}

History of contact with TB patients

Contact with TB patient(s) in household
No
569
Ref
Ref
Ref
Yes
74
$1.24[0.66-2.32]$
0.49
$1.16[0.60-2.27]$
0.66
$1.26[0.54-2.92] \quad 0.60$
Contact with TB patient(s) in the community
No
463
Ref
Ref
Ref
Yes
51
$2.41[1.26-4.62]$
0.01
$1.92[0.93$ - 3.97]
$0.08 \quad 2.02[0.84-4.88]$
0.12

Contact with TB patient(s) at school

Nes $\quad$ No

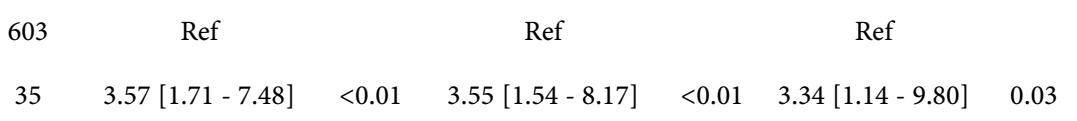

Social activity patterns of adolescents

Encounter with traditional alcoholic beverage drinkers

Never

Yes, a few times in the past

Yes, once to a few times a month

Yes, once a week to more frequently

Closeness to health facility

$$
\begin{aligned}
& \leq 10 \text { mins } \\
& 10-30 \text { mins }
\end{aligned}
$$$$
\geq 30 \text { minutes }
$$

Number of living rooms$$
1
$$

2

Brightness from sunlight in living room(s)

$$
\begin{aligned}
& \text { Very bright } \\
& \text { Somewhat bright }
\end{aligned}
$$

Number of bedrooms

1
2
3
$\geq 4$

107

127

319

95
Ref

$0.33 \quad 0.26[0.09-0.81] \quad 0.02$

$0.13 \quad 0.40[0.09-1.79] \quad 0.23$

$0.03 \quad 6.37[1.84-22.00]<0.01$

Ref

$2.29[1.15-4.56]$

0.02

Ref

Ref
$0.84[0.43-1.62] \quad 0.60$

$3.40[1.67-6.91]$

$<0.01$
$1.91[0.90-4.05]$

$0.091 .51[0.49-4.64]$

0.48

$0.68[0.33-1.41]$

$0.30 \quad 0.35[0.11-1.06] \quad 0.06$

$3.88[1.74-8.67]$

$<0.01 \quad 3.85[1.12-13.20] \quad 0.03$ 


\section{Continued}

Brightness from sunlight in bedrooms

$\begin{array}{lccccccc}\text { Very bright } & 135 & \text { Ref } & & \text { Ref } & & \text { Ref } & \\ \text { Somewhat bright } & 492 & 3.33[1.69-6.58] & <0.01 & 2.89[1.39-6.00] & <0.01 & 2.02[0.74-5.50] & 0.17\end{array}$

Household socioeconomic characteristics

Father's educational attainment

No education

Primary Education

Higher education

Mother's educational attainment

Ref: No education

Primary education

Higher education

Wealth status of household

Richest quintile
Rich quintile
Middle quintile
Poor quintile
Poorest quintile

18

264

276

15

342

215

146

140

142

157

14
Ref

$0.43[0.16-1.21]$

$0.18[0.06-0.52]$

0.11

3.10 [0.34 - 28.07]

$<0.01 \quad 1.66[0.18-15.10]$

Ref

$1.25[0.34-4.54]$

0.74

$1.54[0.38-6.26]$

$0.70[0.19-2.63]$

0.60

$1.13[0.27-4.72]$

$0.55 \quad 2.20[0.23-20.71] \quad 0.49$

$0.87 \quad 1.63[0.17-15.77] \quad 0.67$

COR, crude odds ratio; AOR, adjusted odds ratio; $P$, P-value; Ref, reference; $95 \% \mathrm{CI}, 95 \%$ Confidence Interval; $\geq$, more or equal to; $\leq$, less or equal to; $<$, less than; >, more than. ${ }^{a}$ Unadjusted model; ${ }^{b}$ Model adjusted for wealth index, household and community tuberculosis contact; ${ }^{\mathrm{c}}$ Model adjusted for wealth index, household and community tuberculosis contact, number of living rooms, brightness in the living room and bedroom as well as BMI percentiles.

within a 10-minute walking distance (AOR, 0.30 [95\%CI, 0.13 - 0.69]). Significant associations were also observed between IGRA positivity and living in a house with one living room, living in a house with four or more bedrooms, contact with a TB patient at school and wealth status.

\section{Discussion}

This is the first study to use the IGRA to examine the prevalence of LTBI among in-school adolescents in Tanzania. Encounters with traditional alcoholic beverage drinkers and living environments were identified as risk factors for LTBI, in addition to household socioeconomic status and history of contact with TB patients in the household and community.

The LTBI prevalence rate of $18.1 \%$ identified by IGRA among Tanzanian adolescents 13 - 15 years old is lower than the prevalence rate of $41.7 \%$ reported for those 11 - 15 years old in Cape Town, South Africa [21], and higher than the rate of 3.4\% - 6.2\% reported for those $10-18$ years old in Shanghai, China [12]. These results reflect the order of reported incidence of smear-positive TB, including HIV-positive subjects, per 100,000 population (Tanzania, 306; South 
Africa; 834; and China, 67) [1].

Because most Tanzanian adolescents have received BCG [7], the IGRA assay used in the present study provides a more accurate rate of LTBI in this population than the less-specific TST [26] [27].

LTBI was associated with a history of household contact with a TB patient, but this relationship disappeared after excluding subjects with history of prior TB. These findings suggested most subjects who had lived in the same household as a TB patient, might have later been diagnosed with active TB themselves, as postulated by the WHO [2]. There was also a significant association between LTBI and exposure to MTB in schools, a similar finding to a study conducted in South Africa township which concluded most MTB transmission among adolescents occurs outside of the household, particularly in school [8].

Regarding exposure in the community, the risk of acquiring LTBI was higher among adolescents with than without a history of encounters with traditional alcoholic beverage drinkers. This observation indicated a role of informal gatherings within communities in the spread of MTB. Traditional alcoholic beverage drinking is usually done at gatherings that are associated with ideal environments for MTB transmission like small, overcrowded and poor ventilated traditional bars and contact with drinkers who are active TB patients. These drinkers in turn spread MTB to adolescents with whom they interact informally within the community [28]. This supports the findings from the Malawi study, which used whole-genome sequencing to prove $>50 \%$ of people with MTB had acquired the bacterium from sources other than a TB patient within their households [29].

Living in a house with four or more bedrooms was shown to increase the risk of LTBI, in contrast to previous studies [14] [15]. This may have been partly due to the Tanzania's extended family tradition, whereby those residing in urban areas and in houses with many bedrooms tend to host relatives migrating from rural areas in search of TB treatment services in urban Dar es Salaam [30] [31]. This could result in exposure and transmission of MTB to adolescents living in the host house.

This study also revealed an association between residing in a house located closest to a health facility and IGRA positivity. Residing close to a health facility might increase adolescents' risks for contact with active TB patients who attend those facilities for TB treatment. Consistent with findings from previous studies [14] [15], these results showed the duration of contact with MTB within a shared environment increases the risk of acquiring the bacterium, thus indicating a significant contribution of environmental exposure to MTB to its transmission [8] [29].

Living in a house with two living rooms, compared to one living room, was associated with a lower risk of LTBI. This observation point to an increase in LTBI risk associated with poor ventilation inside the living rooms, supporting reports that overcrowding and poor ventilation increase the risk of acquiring MTB [14] [15]. Our study also showed poor socioeconomic status increased the 
risk of acquiring LTBI, consistent with other studies [15] [28].

Our study had notable strengths including being the first study to use IGRA, rather than TST, to identify LTBI among BCG-vaccinated adolescents in Tanzania. Using IGRA also provided logistic advantages as it does not require a subsequent visit 72 hours from inoculation to interpret the test results, hence convenient and contributes to a lower loss-to-follow-up rate than TST. The TB risk factor questionnaire used ensured ability to identify local LTBI risk factors based on locally available evidence. Also, overall recruitment challenges and participation bias were minimized by avoiding mandatory HIV testing among participants as the test, if were mandatory, would have limited voluntary participation to avoid stigma and discrimination since HIV/AIDS is prevalent in Tanzania [32]. Although HIV/AIDS infection is known to increase the risk of TB [33], this study population was expected to have a low prevalence of HIV of $<6 \%$, as was observed among adolescents 10 - 19 years old in 2014 [34]. Further, free antiretroviral therapy (ART) for HIV-infected children has been available in Tanzania since 2003 [35], hence subjects with HIV on ART would have been identified since all subjects were asked about concomitant medications.

However, our study also had some limitations including omission of a small number of subjects with missing data while conducting the logistic regression analysis, but since their numbers were small, omitting them did not affect our results. In addition, being cross-sectional in nature, it failed to establish causality assumption. Moreover, our study excluded out-of-school adolescents, which limited generalizability of the findings to all adolescents in Tanzania.

\section{Conclusion}

This is the first study to use the IGRA to examine LTBI prevalence among in-school adolescents in Tanzania. Informal social encounters were identified as independent risk factors for LTBI, along with a history of contact with TB patients, living environment characteristics and household socioeconomic status. Efforts focusing on risk of MTB transmission in adolescents at informal social gatherings will improve interventions to reduce LTBI in this population and consequently the subsequent risk of developing active TB disease.

\section{Acknowledgements}

The authors gratefully acknowledge the assistance from Dr. Masashi Kizuki of Tokyo Medical and Dental University in designing the questionnaire. They also thank Dar-PIA team members at Dar-PIA Clinic in Dar es Salaam, Tanzania (Dr. Albert Katana and Dr. Maryam Amour for coordinating meetings at the Ministry of Education and Vocational Training (MoEVT), Ministry of Health, Community Development, Gender, Elderly and Children (MoHCDEC) and President's Office-Regional Administration and Local Government (PO-RALG) as well as organising meetings with schools' administration, Dr. Suleiman Chum, Sr. Mary Ngatoluwa, Sr. Asha Swaleh and Mr. Chijano Makunenge for collecting 
data and Ms. Betty Mchaki for conducting IGRA tests.

The Global Health Innovative Technology Fund (G2015-147) and Japan Society for Promotion of Science (17H02164) supported this work. The funders had no role in study design data collection and analysis, decision to publish, or preparation of the manuscript.

\section{References}

[1] World Health Organization (2016) Global Tuberculosis Report 2016. WHO, Geneva.

[2] World Health Organization (2015) Guidelines on the Management of Latent Tuberculosis Infection. WHO, Geneva.

[3] Getahun, H., Matteelli, A., Chaisson, R.E. and Raviglione, M. (2015) Latent Mycobacterium tuberculosis Infection. New England Journal of Medicine, 372, 127-135. https://doi.org/10.1056/NEJMra1405427

[4] Roy, A., Eisenhut, M., Harris, R.J., Rodrigues, L.C., Sridhar, S., Habermann, S., et al. (2014) Effect of BCG Vaccination against Mycobacterium tuberculosis Infection in Children: Systematic Review and Meta-Analysis. BMJ, 349, g4643. https://doi.org/10.1136/bmj.g4643

[5] von Reyn, C.F. (2017) BCG Vaccination. https://www.uptodate.com/contents/bcg-vaccination

[6] von Reyn, C.F. (2017) Correcting the Record on BCG before We License New Vaccines against Tuberculosis. The Royal Society of Medicine Journals, 110, 428-433. https://doi.org/10.1177/0141076817732965

[7] Ministry of Health and Social Welfare (MoHSW) of Government of Tanzania (2016) WHO and UNICEF Estimates of Immunization: Coverage 2016 Revision. Dar es Salaam, The United Republic of Tanzania: MoHSW, 1-3. http://www.who.int/immunization/monitoring_surveillance/data/tza.pdf

[8] Andrews, J.R., Morrow, C., Walensky, R.P. and Wood, R. (2014) Integrating Social Contact and Environmental Data in Evaluating Tuberculosis Transmission in a South African Township. Journal of Infectious Diseases, 210, 597-603. https://doi.org/10.1093/infdis/jiu138

[9] Tanzania National Bureau of Statistics (NBS) (2016) Tanzania Total Population by District-Regions 2016-2017. Dar es Salaam, The United Republic of Tanzania. http://www.nbs.go.tz/nbstz/index.php/english/statistics-by-subject/population-andhousing-census/844-tanzania-total-population-by-district-regions-2016

[10] Mahomed, H., Hawkridge, T., Verver, S., Abrahams, D., Geiter, L., Hatherill, M., et al. (2011) The Tuberculin Skin Test versus QuantiFERON TB Gold ${ }^{\circledR}$ in Predicting Tuberculosis Disease in an Adolescent Cohort Study in South Africa. PLoS ONE, 6, e17984. https://doi.org/10.1371/journal.pone.0017984

[11] Bianchi, L., Galli, L., Moriondo, M., Veneruso, G., Becciolini, L., Azzari, C., et al. (2009) Interferon-Gamma Release Assay Improves the Diagnosis of Tuberculosis in Children. The Pediatric Infectious Disease Journal, 28, 510-514. https://doi.org/10.1097/INF.0b013e31819abf6b

[12] Hu, Y., Zhao, Q., Wu, L., Wang, W., Yuan, Z. and Xu, B. (2013) Prevalence of Latent Tuberculosis Infection and Its Risk Factors in Schoolchildren and Adolescents in Shanghai, China. European Journal of Public Health, 23, 1064-1069. https://doi.org/10.1093/eurpub/ckt105

[13] Mumpe-Mwanja, D., Verver, S., Yeka, A., Etwom, A., Waako, J., Ssengooba, W., et 
al. (2015) Prevalence and Risk Factors of Latent Tuberculosis among Adolescents in Rural Eastern Uganda. African Health Sciences, 15, 851-860. https://doi.org/10.4314/ahs.v15i3.20

[14] Kashyap, R.S., Nayak, A.R., Gaherwar, H.M., Husain, A.A., Shekhawat, S.D., Jain, R.K., et al. (2014) Latent TB Infection Diagnosis in Population Exposed to TB Subjects in Close and Poor Ventilated High TB Endemic Zone in India. PLoS ONE, 9, e89524. https://doi.org/10.1371/journal.pone.0089524

[15] Lönnroth, K., Jaramillo, E., Williams, B.G., Dye, C. and Raviglione, M. (2009) Drivers of Tuberculosis Epidemics: The Role of Risk Factors and Social Determinants. Social Science and Medicine, 68, 2240-2246. https://doi.org/10.1016/j.socscimed.2009.03.041

[16] Ai, J.-W., Ruan, Q.-L., Liu, Q.-H. and Zhang, W.-H. (2016) Updates on the Risk Factors for Latent Tuberculosis Reactivation and Their Managements. Emerging Microbes \& Infections, 5, e10. https://doi.org/10.1038/emi.2016.10

[17] Nguyen, T.H., Odermatt, P., Slesak, G. and Barennes, H. (2009) Risk of Latent Tuberculosis Infection in Children Living in Households with Tuberculosis Patients: A Cross Sectional Survey in Remote Northern Lao People's Democratic Republic. BMC Infectious Diseases, 9, 96. https://doi.org/10.1186/1471-2334-9-96

[18] National Institute for Health (NIH) (2017) DAR-901 TB Booster Vaccine to Prevent TB in Adolescents (DAR-PIA) Dartmouth-Hitchcock Medical Center. https://clinicaltrials.gov/ct2/show/record/NCT02712424

[19] Von Reyn, C.F., Lahey, T., Arbeit, R.D., Landry, B., Kailani, L., Adams, L.V., et al. (2017) Safety and Immunogenicity of an Inactivated Whole Cell Tuberculosis Vaccine Booster in Adults Primed with BCG: A Randomized, Controlled Trial of DAR-901. PLoS ONE, 12, e0175215. https://doi.org/10.1371/journal.pone.0175215

[20] President's Office Regional Administration and Local Government (PO-RALG) (2016) List of Registered Secondary Schools Datasets 2016 of the United Republic of Tanzania Government Open Data Portal 2016. The United Republic of Tanzania, Dar es Salaam. http://opendata.go.tz/dataset/orodha-ya-shule-za-sekondari-2016

[21] Wood, R., Liang, H., Wu, H., Middelkoop, K., Oni, T., Rangaka, M.X., et al. (2010) Changing Prevalence of Tuberculosis Infection with Increasing Age in High-Burden Townships in South Africa. International Journal of Tuberculosis and Lung Disease, 14, 406-412.

[22] National AIDS Control Program (NACP) (2012) TB Screening Questionnaire for Children above Age 6 and Adult HIV/AIDS Patients. In: National Guidelines for Management of HIV and AIDS, 4th Edition, The United Republic of Tanzania, Dar Es Salaam, 302-304.

https://aidsfree.usaid.gov/sites/default/files/hts_policy_tanzania.pdf

[23] Centers for Disease Control and Prevention (CDC) (2017) National Center for Health Statistics: CDC Growth Charts.

https://www.cdc.gov/growthcharts/clinical_charts.htm

[24] Rutstein, S.O. (2015) Steps to Construct New DHS Wealth Index. https://www.dhsprogram.com/programming/wealth\%20index/Steps_to_constructin g_the_new_DHS_Wealth_Index.pdf

[25] Vyas, S. and Kumaranayake, L. (2006) Constructing Socio-Economic Status Indices: How to Use Principal Components Analysis. Health Policy and Planning, 21, 459-468. https://doi.org/10.1093/heapol/czl029

[26] Trajman, A., Steffen, R.E., Menzies, D., Trajman, A., Steffen, R.E. and Menzies, D. (2013) Interferon-Gamma Release Assays versus Tuberculin Skin Testing for the 
Diagnosis of Latent Tuberculosis Infection: An Overview of the Evidence. Pulmonary Medicine, 2013, Article ID: 601737.

[27] Kleinert, S., Tony, H.P., Krueger, K., Detert, J., Mielke, F., Rockwitz, K., et al. (2012) Screening for Latent Tuberculosis Infection: Performance of Tuberculin Skin Test and Interferon-Gamma Release Assays under Real-Life Conditions. Annals of the Rheumatic Diseases, 71, 1791-1795.

https://doi.org/10.1136/annrheumdis-2011-200941

[28] Schmidt, C.W. (2008) Linking TB and the Environment: An Overlooked Mitigation Strategy. Environmental Health Perspectives, 116, A478-A485. https://doi.org/10.1289/ehp.116-a478

[29] Glynn, J.R., Guerra-Assunção, J.A., Houben, R.M.G.J., Sichali, L., Mzembe, T., Mwaungulu, L.K., et al. (2015) Whole Genome Sequencing Shows a Low Proportion of Tuberculosis Disease Is Attributable to Known Close Contacts in Rural Malawi. PLoS ONE, 10, e0132840. https://doi.org/10.1371/journal.pone.0132840

[30] Wilson, M.E. (1995) Travel and the Emergence of Infectious Diseases. Emerging Infectious Diseases, 1, 39-46. https://doi.org/10.3201/eid0102.950201

[31] Peer, N. (2015) The Converging Burdens of Infectious and Non-Communicable Diseases in Rural-to-Urban Migrant Sub-Saharan African Populations: A Focus on HIV/AIDS, Tuberculosis and Cardio-Metabolic Diseases. Tropical Diseases, Travel Medicine and Vaccines, 1, 6. https://doi.org/10.1186/s40794-015-0007-4

[32] Tanzania Commission for AIDS (TACAIDS), Zanzibar AIDS Commission (ZAC), National Bureau of Statistics (NBS), Office of Chief Government Statistician (OCGS) and ICF International (2013) Tanzania HIV/AIDS and Malaria Indicator Survey 2011-12. Dar es Salaam. https://dhsprogram.com/pubs/pdf/AIS11/AIS11.pdf

[33] Lahey, T., Mackenzie, T., Arbeit, R.D., Bakari, M., Mtei, L., Matee, M., et al. (2013) Recurrent Tuberculosis Risk among HIV-Infected Adults in Tanzania with Prior Active Tuberculosis. Clinical Infectious Diseases, 56, 151-158. https://doi.org/10.1093/cid/cis798

[34] Avert (2016) HIV and AIDS in Tanzania. https://www.avert.org/professionals/hiv-around-world/sub-saharan-africa/tanzania

[35] Bratholm, C., Johannessen, A., Naman, E., et al. (2010) Drug Resistance Is Widespread among Children Who Receive Long-Term Antiretroviral Treatment at a Rural Tanzanian Hospital. Journal of Antimicrobial Chemotherapy, 65, 1996-2000. https://doi.org/10.1093/jac/dkq234 